\title{
B12 deficiency is common in infants and is accompanied by serious neurological symptoms
}

\author{
T Irevall', I Axelsson', E Naumburg (estelle.naumburg@umu.se) ${ }^{1,2}$ \\ 1.Unit of Research, Education and Development, Östersund Hospital, Östersund, Sweden \\ 2.Department of Clinical Science, Paediatrics, Umeå University, Umeå, Sweden
}

\section{Keywords}

Apparent life-threatening event, Infant nutrition, Neurological symptoms, Seizures, Vitamin B12 deficiency

\section{Correspondence}

Estelle Naumburg, Department of Paediatrics,

Östersund Hospital, SE-831 83 Östersund, Sweden.

Tel: +46-63153000

Fax: +46-63154505 |

Email: estelle.naumburg@umu.se

\section{Received}

8 March 2016; revised 22 September 2016; accepted 10 October 2016.

DOI:10.1111/apa.13625

\begin{abstract}
Aim: Adverse neurological symptoms have been linked to vitamin B12 deficiency in infants. This explorative study described the clinical presentation associated with vitamin B12 deficiency in this age group.
\end{abstract}

Methods: The study comprised infants who were born between 2004 and 2012 and were tested for vitamin B 12 levels after they were admitted to a hospital with neurological symptoms at less than one year of age. Vitamin B12 deficiency was defined as low cobalamin in serum and/or increased homocysteine and/or increased methylmalonate. It was diagnosed according to the applicable International Classification of Diseases, 10th revision, and recorded as vitamin B12 deficiency in the medical records. All information was retrieved from medical records and compared to symptomatic infants with normal levels.

Results: Of the 121 infants tested, 35 had vitamin B12 deficiency and 86 had normal levels. Vitamin B12 deficiency was diagnosed at an average age of 1.7 months and was more common among boys. Seizures and apparent life-threatening events were the most common symptoms among infants with B12 deficiency compared to infants with normal levels.

Conclusion: Vitamin B12 deficiency was more common in infants than we expected and presented with severe symptoms, such as seizures and apparent life-threatening events.

\section{BACKGROUND}

Several neurological symptoms have been described in infants with a severe cobalamin deficiency, also referred to as vitamin B12 deficiency. These include irritability, failure to thrive, apathy, anorexia and developmental delay, as well as abnormal electroencephalography and cerebral atrophy on magnetic resonance imaging. The neurological symptoms due to delayed myelination, demyelination, dysmyelination and axonopathy are usually present between the ages of three to nine months (1-4). It can therefore be hypothesised that other neurological symptoms and signs, such as seizures, spasms, apnoea and apparent life-threatening events, can be associated with vitamin B12 deficiency.

Testing for vitamin B12 deficiency is not part of the regular screening process in Sweden when infants present with adverse neurological symptoms and vitamin B12 deficiency have not previously been studied in a defined population in Sweden.

The aim of this explorative study was to describe the first neurological signs and symptoms of infants with vitamin B12 deficiency.

\section{PATIENTS AND METHOD}

The study population included all 11143 children under the age of one, born in Jämtland County between 1 April
2004 and 24 September 2012. We reviewed the medical records for the 121 infants admitted to the only hospital in the county and tested for vitamin B12 and homocysteine in serum and methylmalonate in plasma or serum (Fig. 1).

Infants with no other cause for the symptoms than a vitamin B12 deficiency were described with regard to the first presented symptom. Children with B12 deficiency were defined by low levels of B12 tested by the local laboratory at the hospital (cobalamin $<160 \mathrm{pmol} / \mathrm{L}$ ) and/or increased total homocysteine $(>10 \mu \mathrm{mol} / \mathrm{L})$ and/or increased methylmalonate, together with ICD-10 codes E53.8, E53.9, D51.3

\section{Key notes}

- Adverse neurological symptoms have been linked to vitamin B12 deficiency in infants, and this explorative study described the clinical presentation associated with vitamin B12 deficiency in this age group.

- Vitamin B12 deficiency was diagnosed at an average age of 1.7 months and was more common in infants with neurological symptoms $(35 / 121)$ than we expected.

- The deficiency was presented with severe symptoms such as seizures and apparent life-threatening events. 


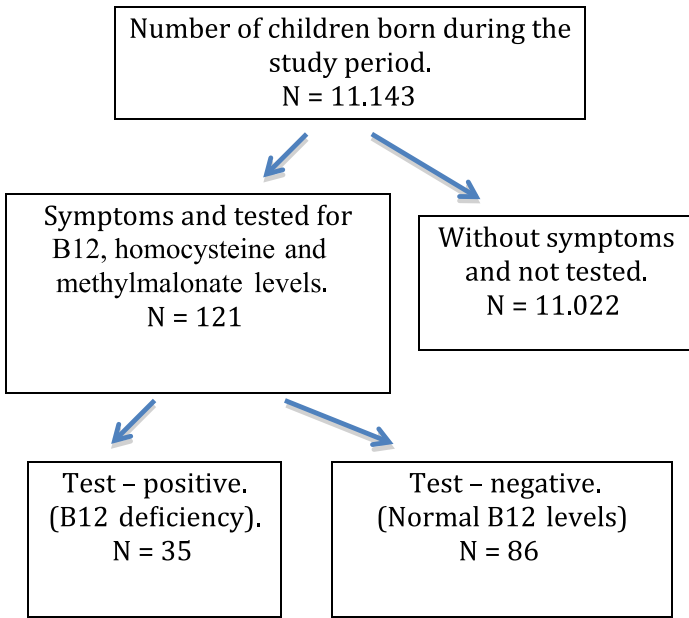

Figure 1 Number of children included in the study.

D51.8 or D51.9 and stated as vitamin B12 deficiency in medical records. B12, homocysteine and methylmalonate were measured at the same time. The tests were carried out at the local hospital, except for methylmalonate, which was analysed at different laboratories during the time period and was defined as a plasma- or serum level of either $<0.37 \mu \mathrm{mol} / \mathrm{L}$ or $<0.4 \mu \mathrm{mol} / \mathrm{L}$. Each case was defined according to the normal levels defined by the available laboratory. Infants admitted to the hospital with symptoms typical of a vitamin B12 deficiency, but with normal levels and no obvious cause for the symptoms, were presented for comparison.

Information on symptoms and age in months at diagnosis and treatment was retrieved from medical records. Each infant could present with more than one symptom. An electroencephalography (EEG) was not available for all patients and therefore not included in research data.

All analyses were descriptive in relation to B12 deficiency or normal levels.

This nonintervention study was a medical students' project carried out as part of the medical programme at Umeå University, Sweden, and involved extracting information from medical records at a local hospital. According the Central Ethical Review Board in Sweden, and the law on ethics in research, ethical approval by the local ethics board was not necessary (5).

\section{RESULTS}

We study hospital had tested 121 children who were less than one year of age for vitamin B12 levels during the study period and found that 35 infants had B12 deficiency and 86 had normal levels (Fig. 1, Table 1). B12 deficiency was defined in 10 infants $(29 \%)$ by low levels of vitamin B12, 26 infants (74\%) by increased homocysteine and/or 27 infants (77\%) with increased methylmalonate (Table 2). The diagnosis of B12 deficiency was decided by the clinician if only a single abnormal level

\begin{tabular}{|c|c|c|c|}
\hline & B12 deficiency & Normal levels & Total \\
\hline Number (M/F) & $35(21 / 14)$ & $86(39 / 47)$ & $121(60 / 61)$ \\
\hline Symptom (months) & $1.7(0-10 \mathrm{~m})$ & $2.7(0-11 \mathrm{~m})$ & \\
\hline Diagnose (months) & $3(0-13 \mathrm{~m})$ & & \\
\hline \multicolumn{4}{|c|}{ Symptoms (more than one/infant) } \\
\hline Seizures and spasm & $16(45 \%)$ & $26(30 \%)$ & $42(35 \%)$ \\
\hline Apnoea & $8(23 \%)$ & $16(19 \%)$ & $24(20 \%)$ \\
\hline ALTE & $5(14 \%)$ & $4(5 \%)$ & $9(7 \%)$ \\
\hline Other neurological* & $8(23 \%)$ & $29(34 \%)$ & $37(31 \%)$ \\
\hline Other ${ }^{\dagger}$ & $9(26 \%)$ & $30(35 \%)$ & $39(32 \%)$ \\
\hline
\end{tabular}

Table 2 B12, homocysteine and methylmalonate in the group of children with defined vitamin B12 deficiency

\begin{tabular}{lccc} 
& \multicolumn{2}{c}{ Number } & \\
\cline { 2 - 3 } Test & Total & $(M / F)$ & Mean (min-max) \\
\hline B12 & & & \\
$\quad$ Decreased & 10 & $6 / 4$ & $136.2(104-159)$ \\
$\quad$ Normal $(>160 \mathrm{pmol} / \mathrm{L})$ & 25 & $15 / 10$ & $233.7(165-317)$ \\
Homocysteine & & & \\
$\quad$ Increased & 26 & $16 / 10$ & $14.4(10-29)$ \\
$\quad$ Normal $(<10 \mu \mathrm{mol} / \mathrm{L})$ & 9 & $5 / 4$ & $7.1(4-9)$ \\
Methylmalonate & & & \\
$\quad$ Increased & 27 & $17 / 10$ & \\
$\quad$ Normal* & 3 & $0 / 3$ & \\
$\quad$ Missing & 5 & $4 / 1$ & \\
\hline
\end{tabular}

*slight differences of cut-off values from different laboratories.

was found, but the clinical picture was typical: in one infant with a low B12 level, in three infants with increased homocysteine and in seven infants with increased methylmalonate.

B12 deficiency was more common among boys (63\%). Infants with B12 deficiency were younger and were tested earlier than infants with normal levels (Table 1). Severe neurological symptoms, such as seizures, apnoea and ALTE, were more common among infants with B12 deficiency, while other neurological or unspecific symptoms were more common among infants with normal levels (Table 1).

All infants, but one, with vitamin B12 deficiency were treated with intramuscular injections of $0.5-1.0 \mathrm{mg}$ vita$\min$ B12. In the other case, a nutritionist advised the parents on improved nutrition for their infant. The majority of the infants (77\%) only needed one injection of that dose, but seven (20\%) infants needed two injections to increase the level of vitamin B12 to normal. When they were followed up at 12-18 months of age, all infants had normal levels of vitamin B12, with no adverse complications as a result of the treatment and with normal neurological development. 


\section{DISCUSSION}

We found that vitamin B12 deficiency was much more common than expected and the estimated incidence in this study was at least 35 of 11143 or 314 of 100000 and probably higher because only a small fraction of the infants in the population had been tested. This was in line with a recent Norwegian study (6). The incidence of B12 deficiency varies across the world and tends to be high in rural and vegetarian populations (3,7-9). Our findings, along with the Norwegian study (6), indicated that B12 deficiency can be relatively common in other populations as well.

Low levels of vitamin B12 and high levels of homocysteine and methylmalonate are seen between birth and six months of age, suggesting an impairment in cobalamin function (10). Normal mean levels of vitamin B12 of 217$314 \mathrm{pmol} / \mathrm{L}$ in the age group of four days to six months have been described (10). In our study, we included levels of vitamin B12, homocysteine, methylmalonate as well as the clinical picture in the definition of infants with B12 deficiency. Our study did not adjust vitamin B12 levels to age, which may have introduced a risk of misclassification. However, our cut-off point (160 pmol/L) was well below the published normal range in this age group of 217-314 pmol/L (10). The level of methylmalonate was analysed at different laboratories, which may have introduced incorrect interpretation of the data, but the differences between the cut-off values were small. Methylmalonate levels are described to be higher in infants compared to older children and adults. Thus, age-specific reference levels are needed. However, age adjustments and different laboratories accounted for infants with B12 deficiency as well as infants with normal levels, and thus, the risk of selection bias was reduced.

Severe vitamin B12 deficiency in infants may produce several neurological symptoms $(2,6,11)$. In our study, severe symptoms, such as seizures and apparent life-threatening events, were more common among infants with B12 deficiency than infants with normal levels (Table 1). The underlying mechanisms of neurological symptoms involve delayed myelination or demyelination of nerves (2). The rapid response to supplements in terms of improvement of neurological symptoms as well as B12 levels in our study was in line with other studies and published case reports $(1,6)$. An early diagnoses and start of treatment are therefore crucial.

The B12-deficient infants were treated with 1-2 intramuscular injections of $0.5-1.0 \mathrm{mg}$ vitamin B12. No adverse reactions were noted, but it may be better to start with smaller doses and increase them over several days (12). An association between neonatal vitamin B12 deficiency following maternal inadequate intake or malabsorption of vitamin B12 has been described in some studies, especially among vegan mothers and children who are exclusively breastfed, while others could not prove this association (13-17). Information on maternal food preference was not included in the medical records and this limited the study. According to a Swedish survey, about $10 \%$ of the population are vegetarians or vegans and this is more common in the larger cities and in the south of Sweden (18). We believe that in Jämtland, in Northern Sweden, the vast majority of women eat meat. Most infants (63\%) are exclusively breastfed until the age of six months in Sweden (19). The number of infants exclusively breastfed in the study population was unknown, as there was no information on breastfeeding in the medical records. Therefore, we do not know whether the unexpectedly high number of infants with vitamin B12 deficiency in the county of Jämtland was due to dietary causes.

The low number of cases and the retrospective collection of data limited the study. However, this study was based on all infants admitted to the only hospital in the county, which reduced the risk of selection bias.

Most infants with B12 deficiency in our study presented with symptoms at an earlier age, an average of 1.7 months, compared to other studies that described B12 deficiency at the ages of four to ten months $(2,13,14)$. However, in our study, all infants were tested before the age that infants normally present with the lowest level of vitamin B12 during childhood $(10,15)$. There was no follow-up on biochemical cobalamin function among infants with normal levels and, therefore, it is not known whether these infants presented with lower levels later in life.

This study, as well as other studies, suggests that early diagnosis and treatment are beneficial to infant development $(3,6,20)$. Prolonged low levels of vitamin B12 have been linked to neurological symptoms $(1,14,21)$. The association between vitamin B12 levels and the type of neurological symptom, as well as long-term beneficial effects of early treatment, has not been studied. Our data could stimulate similar studies in larger populations by other research groups and may lead to changes in recommendations for screening and prevention of B12 deficiency and diagnostic evaluation of neurological symptoms in infants.

\section{CONCLUSION}

Our study confirmed recent studies that vitamin B12 deficiency was much more common in infants than previously described. Furthermore, severe neurological symptoms, such as seizures and apparent life-threatening events, were common in young infants with a B12 deficiency.

\section{ACKNOWLEDGEMENTS}

We thank all the children in this study and Kristina Söderström who provided language editor services on behalf of Mid Sweden University.

\section{FUNDING}

This study did not receive any external funding.

\section{CONFLICTS OF INTERESTS}

The authors declare that they have no competing interests. 


\section{References}

1. von Schenck U, Bender-Gotze C, Koletzko B. Persistence of neurological damage induced by dietary vitamin B-12 deficiency in infancy. Arch Dis Child 1997; 77: 137-9.

2. Dror DK, Allen LH. Effect of vitamin B12 deficiency on neurodevelopment in infants: current knowledge and possible mechanisms. Nutr Rev 2008; 66: 250-5.

3. Rasmussen SA, Fernhoff PM, Scanlon KS. Vitamin B12 deficiency in children and adolescents. J Pediatr 2001; 138: $10-7$.

4. Fine EJ, Soria E, Paroski MW, Petryk D, Thomasula L. The neurophysiological profile of vitamin B12 deficiency. Muscle Nerve 1990; 13: 158-64.

5. Lag (2003:460) om etikprövning av forskning som avser människor, 2003.

6. Torsvik I, Ueland PM, Markestad T, Bjorke-Monsen AL. Cobalamin supplementation improves motor development and regurgitations in infants: results from a randomized intervention study. Am J Clin Nutr 2013; 98: 1233-40.

7. Hunt A, Harrington D, Robinson S. Vitamin B12 deficiency. BMJ 2014; 349: g5226.

8. Allen LH. How common is vitamin B-12 deficiency? Am J Clin Nutr 2009; 89: 693S-6S.

9. Garcia-Casal MN, Osorio C, Landaeta M, Leets I, Matus P, Fazzino F, et al. High prevalence of folic acid and vitamin B12 deficiencies in infants, children, adolescents and pregnant women in Venezuela. Eur J Clin Nutr 2005; 59: 1064-70.

10. Monsen AL, Refsum H, Markestad T, Ueland PM. Cobalamin status and its biochemical markers methylmalonic acid and homocysteine in different age groups from 4 days to 19 years. Clin Chem 2003; 49: 2067-75.

11. Duggan C, Srinivasan K, Thomas T, Samuel T, Rajendran R, Muthayya S, et al. Vitamin B-12 supplementation during pregnancy and early lactation increases maternal, breast milk, and infant measures of vitamin B-12 status. J Nutr 2014; 144 : 758-64.

12. Demir N, Koc A, Ustyol L, Peker E, Abuhandan M. Clinical and neurological findings of severe vitamin B12 deficiency in infancy and importance of early diagnosis and treatment. J Paediatr Child Health 2013; 49: 820-4.

13. Naver L, von Dobeln U, Hagenfeldt L. B12 deficiency in breast-fed children. Maternal deficiency may cause severe symptoms in children. Läkartidningen 1995; 92: 3331-4.

14. Honzik T, Adamovicova M, Smolka V, Magner M, Hruba E, Zeman J. Clinical presentation and metabolic consequences in 40 breastfed infants with nutritional vitamin B12 deficiencywhat have we learned? Eur J Paediatr Neurol 2010; 14: 48895.

15. Hay G, Johnston C, Whitelaw A, Trygg K, Refsum H. Folate and cobalamin status in relation to breastfeeding and weaning in healthy infants. Am J Clin Nutr 2008; 88: 105-14.

16. Larsen PS, Nybo Andersen AM, Uldall P, Bech BH, Olsen J, Hansen AV, et al. Maternal vegetarianism and neurodevelopment of children enrolled in The Danish National Birth Cohort. Acta Paediatr 2014; 103: e507-9.

17. Koc A, Kocyigit A, Soran M, Demir N, Sevinc E, Erel O, et al. High frequency of maternal vitamin B12 deficiency as an important cause of infantile vitamin B12 deficiency in Sanliurfa province of Turkey. Eur J Nutr 2006; 45: 291-7.

18. Djurens rätt. Demoskopundersökning: Fyra av tio kan tänka sig äta vegetariskt av hänsyn till djuren: Djurens rätt. Available at: http://www.mynewsdesk.com/se/ djurens_ratt/pressreleases/demoskopundersoekning-fyra-avtio-kan-taenka-sig-aeta-vegetariskt-av-haensyn-till-djuren394192 (accessed on April 12, 2010).

19. Official Statistics of Sweden. Breast-feeding and smoking habits among parents of infants born in 2010. Statistics Health and Medical Care, 2012. Available at: http:// www.socialstyrelsen.se/ (accessed on August 29, 2012).

20. McPhee AJ, Davidson GP, Leahy M, Beare T. Vitamin B12 deficiency in a breast fed infant. Arch Dis Child 1988; 63: 921-3.

21. Graham SM, Arvela OM, Wise GA. Long-term neurologic consequences of nutritional vitamin B12 deficiency in infants. J Pediatr 1992; 121: 710-4. 